\title{
LA NUEVA HISTORIA LITERARIA DE COLOMBIA: PREOCUPACIONES Y PROYECCIONES
}

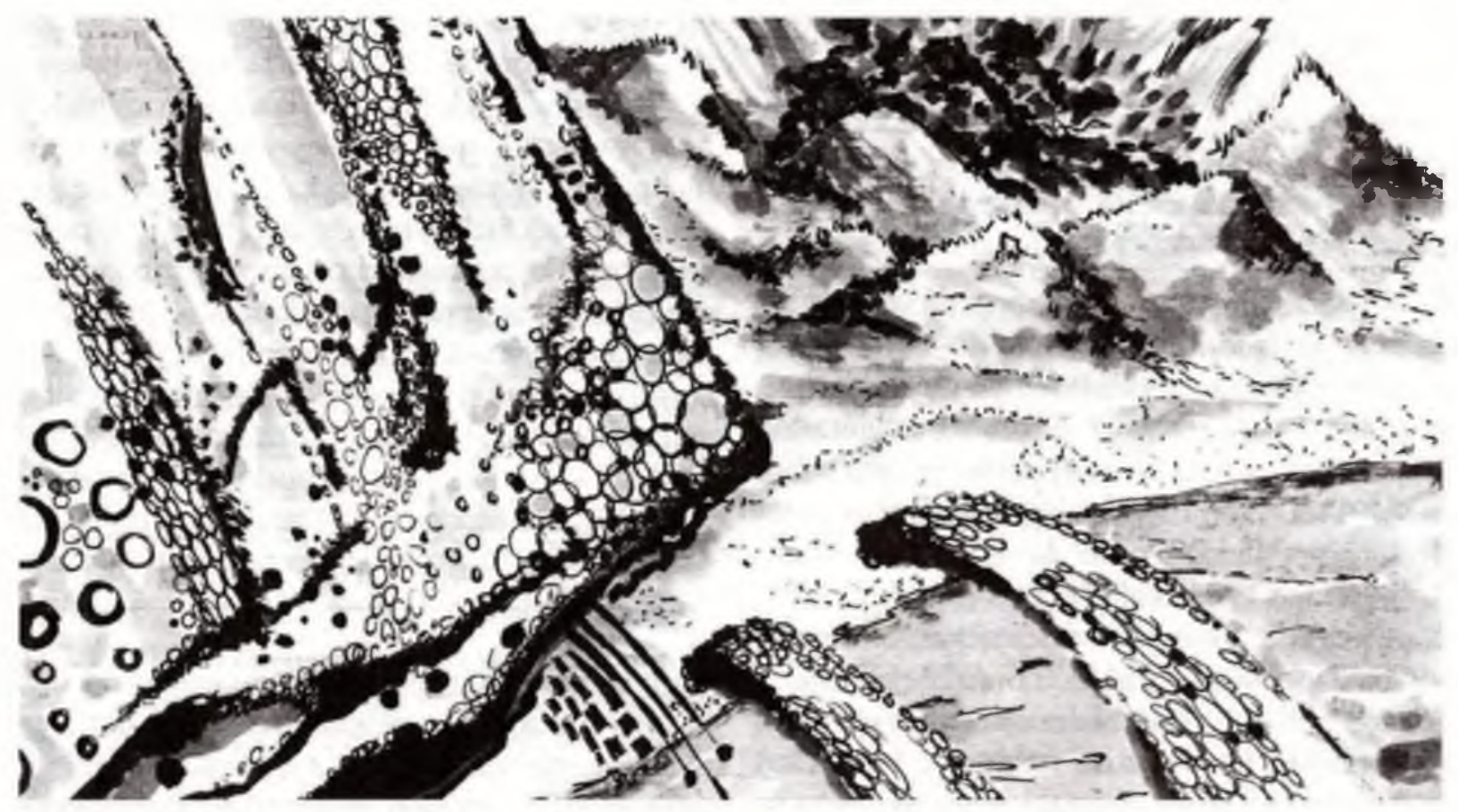

LEONARDO AUGUSTO MONROY Z. MAGÍSTER EN LITERATURA HISPANOAMERICANA PROFESOR INVESTIGADOR UNIVERSIDAD DEL TOLIMA leomonroyz@gmail.com

\begin{abstract}
In search of autonomy in the remaking of the literary field in Colombia, many Colombian scholars believe they can give another meaning to the literary history of the country. This can be made through a careful and right approach to the nation, canon, periodization and reception concepts of literature and literary institution. Doing this will redound to the benefit of both the configuration of a clearly-defined national literary identity and the teaching task using the literary productions.
\end{abstract}

\section{KEY WORDS}

literary history, nation, canon, literary reception

\section{CINCO PREOCUPACIONES BÁSICAS DE LA NUEVA HISTORIA}

\section{LITERARIA DE COLOMBIA}

D uede resultar paradójico que unas décadas después de que parte de la investigación europea en ciencias sociales haya declarado la disolución de las disciplinas, y entre ellas la de la historia, hoy esté creciendo en el país la reflexión sobre la historia de la literatura colombiana "con tanta intensidad como en aquel siglo XIX de incipiente independencia" (Vallejo, 2007). Las razones de esta asimetría entre las problemáticas presentadas en Europa y las colombianas - por lo menos en el campo de la historia literaria - pueden tener su génesis en el intento de autonomía para la generación de nuestros propios problemas de investigación, 
métodos y conceptos, planteados entre otros por Ángel Rama, Antonio Cándido y Alejandro Losada (D'Allemand, 2003), en la relectura de los textos de Rafael Gutiérrez Girardot (Acosta, 2003), en una creciente inconformidad por los logros de las historias de la literatura nacional que hasta la fecha se han escrito en Colombia, o incluso en la necesidad que se le plantea a los estudiosos colombianos de reelaborar una nueva imagen de nuestra literatura, en vísperas del bicentenario de la independencia de la nación.

Cualquiera que sea la razón, en Colombia ya hay un monto suficiente de expresiones académicas que permiten emprender la elaboración de su nueva historia de la literatura (Bedoya, 2007) La preocupación teórica sobre la historia literaria, consignada en revistas de universidades como la Nacional, la de Antioquia, o la del Valle, son el primer eslabón para, desde nuestras discusiones internas, seguir la ruta de países como Argentina y Ecuador, en los que actualmente se re- escriben historias de la literatura nacional. En dichas publicaciones universitarias- y en los eventos en los que se ha discutido sobre la cuestión - se evidencia el esfuerzo por reconsiderar, entre otros, los conceptos de nación, canon, periodización, recepción de la literatura y la institución literaria, que amplíen y renueven la escritura de la historia literaria.

En el caso del concepto de nación, desde 1970, y especialmente bajo las propuestas de Ángel Rama, Antonio Cornejo y Alejandro Losada, se viene revisando la idea de nación construida en el siglo XIX en Latinoamérica, en beneficio de una concepción más incluyente de las particularidades culturales que conforman el mapa interno de los países. Conceptos como el de heterogeneidad y totalidad contradictoria (Cornejo), transculturación (Rama) o procesos regionales particulares (Losada), constituyen las respuestas al "carácter problemático de la Literatura Nacional" en el que existen, entre otras, una "ficción de unidad y de evolución lineal y cohesión de los procesos culturales", "limitaciones para deslindar literaturas ilustradas y populares" e "incapacidad para aprehender variantes intranacionales" (D'Allemand, 2003, 88)
Estas nuevas perspectivas son retomadas en Colombia, entre otros, por un libro como Literatura y Cultura (2000), en cuyo estudio preliminar se explicita la abierta tendencia multicultural que define la antología de textos críticos incluidos en su contenido. La revaluación del concepto de nación, desde una mirada que privilegie la diversidad en la unidad, lleva a la reflexión no solo de las metodologías y periodizaciones realizadas en cada tradición particular (de las varias que se incluyen en el país) sino que pone en escena la posibilidad de abordar objetos de estudio mucho más específicos: las literaturas regionales (Henao, 2006), supraregionales (tales como la Caribe, o la Andina), o departamentales, así como también las que, por razones étnicas y/o lingüísticas, no han sido del todo incluidas en el marco de la historia de la literatura colombiana (las literaturas negras e indígenas, por ejemplo) (Vargas, 2006)

Frente a las posibilidades de desarrollo de una historia literaria que delimite más estrictamente el ámbito de estudio - en especial en términos territoriales - existen reticencias de quienes consideran que sólo es posible hablar de buena - mala literatura, sin consideraciones del lugar de donde provenga, o de aquellos que asumen los estudios intraregionales como un ejercicio de fragmentación e incomunicación del continente, que en apariencia tiene problemáticas comunes.

En contraposición a estas recriminaciones, Ángel Rama ha formulado una propuesta plausible sobre los procesos literarios de una región determinada. En su texto García Márquez. Edificación de un arte nacional y popular, el investigador uruguayo afirma que "no habría modo de comprender la realidad hispanoamericana si no comenzáramos por reconocer la existencia de áreas culturales independientes" (Rama, s.f., 11) El área cultural no se corresponde necesariamente con las delimitaciones político administrativas, sino que se concibe como un "fondo étnico - cultural" que tiene unos "elementos históricos determinantes que se han ido acumulando" (Rama, s.f., 12) El concepto de área cultural y el diálogo complejo que entabla el escritor con ella, puede servir de salida a los conflictos que se presentan cuando se parcelan zonas internas de la nación, tales 
como los del esencialismo (se estudia un escritor porque nació en una determinada región, aunque haya migrado a temprana edad), o el desconocimiento de voces que, sin haber nacido en un área cultural, han participado de ciertos procesos histórico - culturales que derivan en un sentido de pertenencia.

Una segunda reconsideración en el marco académico colombiano se realiza al concepto de canon. Toda vez que la posibilidad de construcción de una nueva historia de la literatura colombiana parte no ya de principios estrictamente literarios, sino del concepto de nación, o de área cultural asociado a las prácticas artísticas, se implementa una cierta flexibilidad frente a los criterios estéticos modernos, en beneficio de una comprensión más amplia de la diversidad cultural decantada en las obras. La parcelación de la perspectiva en sectores de la realidad cada vez más específicos (como las regiones o los departamentos), hace más difícil hallar cánones universales, pero en cambio puede ser la base para interpretaciones de un material literario aun no abordado, o incluso de aquellos que habitualmente no han sido considerados literarios y que bajo perspectivas actuales - como las de los estudios culturales - son aceptados actualmente como objetos de reflexión ${ }^{80}$.

Dos miradas antagónicas frente a este fenómeno se pueden apreciar en los textos de Germán Patiño y de Olga Vallejo Murcia: en el primero de ellos se defiende la construcción de una historia de la literatura basada estrictamente en lo literario, es decir, una historia en la que no se llegue a perder el objeto de estudio específico de la literatura (lo literario) (Patiño, 2004), mientras que en el segundo caso, la profesora Vallejo lidera una investigación denominada Tradiciones de la palabra que "no implica un rechazo a la concepción antológica de literatura sino más bien la convivencia de la palabra impresa, la palabra escrita, la palabra dicha, la palabra actuada, la palabra femenina, negra e indígena, la palabra de la prensa, del discurso y del ensayo" (Vallejo, 2007)

Así mismo, esta nueva actitud frente al canon desvertebra la mirada que sólo se fija en las figuras reconocidas históricamente por la crítica y la historia literarias. En este sentido, varios de los artículos generados en la Universidad de Antioquia - y algunos de la universidad Nacional - apuntan a señalar una suerte de esnobismo frente a las figuras representativas impuestas en el pasado, la falta de legitimidad de los criterios utilizados por quienes han establecido el grupo de obras cumbres de la nación y la necesidad de hallar nuevas piezas literarias que expresen con mayor amplitud la particularidad de las sociedades de un momento determinado de nuestra historia.

No es suficiente entonces con la exploración de los hitos de nuestra literatura - que por demás tienen un número reducido -, sino analizar las formas de expresión y las maneras como se reelabora el mundo, más allá de Jorge Isaacs, José Eustasio Rivera, Gabriel García Márquez y Álvaro Mutis, para acudir solo a la clasificación hecha por Luz Mary Giraldo (1994). No quiere esto decir que dichos autores - y las obras que componen su repertorio -, deban dejarse en el olvido, sino que los estudios que hacen su relectura, conviven con los que comprenden un cuerpo más amplio de producciones literarias.

Otro de los ejes sobre los que ha derivado la discusión, es el problema de la periodización. La inconformidad frente a experiencias pasadas, ha despertado una búsqueda por nuevas fórmulas para entender los procesos literarios de la nación multicultural: ni el "diccionario de nombres o enciclopedia de fechas" (Bedoya, 2006), ni la organización por generaciones o por décadas, ni las etapas subsumidas en etiquetas literarias que en muchas ocasiones se aplican sin una reflexión crítica (clasicismo, romanticismo, modernismo, etc.), parecen satisfacer las expectativas de la academia colombiana reciente.

Al respecto, y ya desde hace unas décadas atrás, el profesor Rafael Gutiérrez Girardot había reflexionado sobre la cuestión, y con base en la lectura de Pedro Henríquez Ureña, llegaba a la conclusión de que tal vez no es necesaria una delimitación a priori de los periodos (Gutiérrez, 1986, 45), sino observar los momentos en los que, con el cambio de una sociedad específica, 
existe también un cambio en las formas de expresión literarias (Gutiérrez, 1986, 32). Las reacciones a esta propuesta de periodización, critican la dependencia del discurso literario frente al devenir social, en una aparente pérdida de autonomía de la propia evolución de la literatura.

El estudio "Los comienzos de la novela colombiana en el siglo XX" de la profesora Patricia Trujillo, reflexiona sobre la variedad de criterios de periodización que han realizado algunos historiadores de la literatura colombiana, en especial en el género novelesco. Una de sus conclusiones es que, a la propuesta de periodización centrada en los cambios sociales, se opone otra netamente estética. Para la investigadora, la salida es pensar en "la combinación de los criterios de periodización anteriormente mencionados" (Trujillo, 2007, 256)

Una tercera mirada sobre la periodización, es la que implícitamente se expone en el libro Literatura y cultura y que teoriza Gustavo Adolfo Bedoya en "La periodización de la literatura colombiana en sus historias literarias". De acuerdo con el autor, una salida a los problemas de periodización es - tal como se hace en Literatura y cultura - "la división del material en grandes temas literarios" (Bedoya, 2006). Los tres planteamientos - Rafael Gutiérrez, Patricia Trujillo, Gustavo Bedoya deben debatirse, e incluso se debe revisar la pertinencia de uno u otro en el contexto de experiencias investigativas de áreas culturales específicas. El problema de la periodización es uno de los que tal vez está en mora de ser mayormente abordado y sólo a través de indagaciones que apliquen principios teóricos, es posible evaluar la validez de las propuestas.

Una cuarta preocupación de la academia frente a la formulación de principios para la elaboración de una nueva historia de la literatura colombiana, es la posibilidad de pasar de una historia de libros y autores a una historia de la lectura y las apropiaciones de los textos. La profesora Carmen Elisa Acosta ha hecho énfasis en Colombia en esta línea de indagación, y retomando los más recientes exponentes de la escuela de los Annales francesa - especialmente a Roger Chartier - tiene ya escritos varios artículos en los que se aplica esta perspectiva (Acosta, 2005) . La propuesta implica desplazar el eje de atención, desde la exploración de los sentidos de las obras - realizada por la crítica literaria habitualmente - a la consideración de las maneras como varias capas de la población se apropian de los textos. Dichas investigaciones - como lo muestra el mismo Chartier - requieren de una visita a fuentes diferentes a las que convencionalmente tiene el investigador, que implican, entre otras, además de las marcas que aparecen en las mismas obras literarias, la lectura de la llamada subliteratura, y de textos que no necesariamente entren en el contexto de lo literario, la exploración de otras expresiones artísticas - como la pintura - y la profundización en archivos de prensa y en las publicaciones de la época, en los que se explicite la manera como grupos diferentes, interpretaron y aprehendieron una obra literaria ${ }^{81}$.

Cercano a esta propuesta de elaboración de una historia de la lectura, se encuentra el profesor Hubert Poppel, quien acudiendo a la academia alemana, habla de una "historia de la recepción" que tiene tres pasos fundamentales: una línea diacrónica que ubique una obra determinada en un proceso de rupturas y que, de igual manera, observe las diferentes críticas que se han hecho de ella; una línea sincrónica, que ponga la obra en el contexto de otros discursos contemporáneos a su aparición; y una tercera mirada a la "función social de la literatura" en tanto expresión que puede llegar a transformar las visiones de mundo del lector $(2003,15)$. Es en especial en este tercer llamado, en el que la propuesta del profesor Poppel - que no parece haber tenido desarrollo en Colombia - se acerca a la de la profesora Carmen Elisa Acosta. Como quiera que sea, estas dos líneas teóricas plantean retos a la formulación de la historia de la literatura colombiana que quiera desprenderse del análisis clásico centrado en obras y autores.

81 Una de las diferencias entre la propuesta de Roger Chartier y la de la profesora Carmen Elisa Acosta es que en esta última hay un cuidado por delimitar las formas de apropiación a las expresiones literarias y no - como lo hace el investigador francés - a los libros en general. 
El quinto y último interrogante frente a la investigación en la historia de la literatura colombiana se concentra en la exploración de la llamada institución literaria, a través de la lectura de publicaciones culturales que han aparecido en un periodo de la historia de un área cultural específica. El concepto de institución literaria parte de Rafael Gutiérrez Girardot, como una preocupación por observar los elementos que median entre la obra y la sociedad. Al realizar un barrido por los intelectuales que se dedicaron a dar cuenta de la relación entre literatura y sociedad, el profesor colombiano percibe que pese a que Marx y Hegel no se refirieron a una relación de reflejo, la lectura hecha por Lenin a Marx no permitió a los estudios de historia social de la literatura huir de esa concepción, en una tradición que, de acuerdo con Gutiérrez Girardot, va hasta Lukács y Goldmann (Gutiérrez, s.f.)

Es el mismo profesor colombiano quien ve en la propuesta de Walter Benjamin una disposición nueva de la mediación entre la literatura y la sociedad: Benjamin traza el camino en el que, para entender la literatura no se debe plantear únicamente la pregunta de lo que ella es en esencia, sino del lugar que la literatura ocupa como institución en el marco de las otras instituciones que conforman la sociedad (Gutiérrez, 1976)

La importancia de Benjamin para la sociología de la literatura es, entre otras, metodológica, y radica en que una vez el analista del arte entiende que los géneros, los sentidos y las formas de expresión, es decir, las obras literarias en su particularidad, están influidas y casi que determinadas por la institución literaria, puede y en el caso de Rafael Gutiérrez, debe - recurrir a la investigación de las formas materiales de esa institución, que "abarca los productores de la literatura, las editoriales y los escritores, los medios de difusión de la literatura, esto es, bibliotecas, librerías, revistas, salones literarios, estudios literarios en colegios y universidades, salas de lectura, y los diversos grupos de lectores, esto es, la llamada recepción de la literatura" (Gutiérrez, 2001, 117), así como también, las preferencias en temáticas y modelos literarios de ciertos momentos de la literatura (Gutiérrez, 1986, 37)
Es bajo ese concepto de institución literaria como se entienden, de manera más certera, y de acuerdo con el autor de Aproximaciones, los diferentes procesos que unen la obra literaria con la sociedad en la que se inscribe. Rafael Gutiérrez plantea que para la elaboración de una historia de la literatura se deben tener en cuenta entonces dos momentos que revisten la misma importancia para el investigador: el primero, el análisis de la institución literaria que ilumine algunos aspectos de la vida cultural en la que nace una obra, y el segundo, el de la interpretación de la obra en su configuración interna.

Las cinco preocupaciones que aquí se han presentado, se convierten en preguntas para quienes deseen asumir desde nuevas perspectivas, la cuestión de los procesos literarios en el país. Ampliando la cuestión, toda vez que un cambio en uno de los enfoques para abordar la literatura - en este caso el historiográfico - ejerce resonancia sobre los demás - crítico, teórico y pedagógico - a continuación se explorarán las posibles consecuencias que estas nuevas consideraciones en la historiografía literaria pueden acarrear en el país, específicamente en el ámbito de la enseñanza de la literatura.

\section{POSIBLES PROYECCIONES EN EL ÁMBITO DE LA ENSEÑANZA}

En las conclusiones de un ejercicio de investigación reciente, la profesora Olga Vallejo expresó a través de un artículo las relaciones entre la historiografía literaria tradicional y la enseñanza de la literatura en Colombia (Vallejo, Papel Periódico). La aceptación acrítica del modelo de diccionario de obras y autores, llevó a las historias didácticas de la literatura colombiana, a algunos manuales para colegios y a los libros de texto, a presentar lecturas superficiales de los procesos literarios. El resultado concreto en la formación de los estudiantes fue la obsesión por la memorización 
de datos inconexos, en los que la obra no se interpelaba y la interpretación era desdeñada.

No se puede negar que algunos de esos vicios continúan latentes en la enseñanza en los colegios, en donde en ocasiones, aun se trabaja con Manuales de la Literatura Colombiana obsoletos, o con los análisis gramaticales para las obras literarias (Ver Rodríguez, 1991), y en general, con perspectivas distantes de las reflexiones hechas en los niveles académicos superiores. Sin embargo, la decisión actual dentro de quienes éticamente piensan en el fenómeno de lo literario desde la docencia, es explorar las posibilidades significativas de las obras desde un enfoque crítico - derivado del estructuralismo, la hermenéutica, la sociocrítica, etc. - en la mayoría de los casos, foráneo.

Es una decisión entendible - teniendo en cuenta las experiencias del pasado - y que puede llevar a excelentes resultados en la interpretación literaria, pero para no olvidar los procesos históricos, una implementación más fina de las nuevas tendencias de la historia literaria colombiana, puede mostrar caminos promisorios para la enseñanza de la literatura tanto en los colegios como en las universidades. Inspeccionaré a continuación algunas de las posibilidades de entronque entre una posible nueva historia literaria de Colombia y enseñanza de la literatura.

La superación de ese viejo modelo de periodización en el que, como ya se ha indicado en líneas anteriores, se presenta una lista de obras y autores, debe generar unas posibilidades de conocimiento diferente: bajo la interpretación de las relaciones de la obra con su momento y espacio, los estudiantes pueden entender de manera menos superficial las implicaciones que una o varias producciones tuvieron no sólo en la tradición literaria sino en la construcción y/o deconstrucción de imaginarios culturales y formas de ver el mundo. Se trata de configurar un conocimiento más amplio sobre los procesos que llevaron a una literatura - sus lenguajes, sus lectores, sus cosmovisiones- y a la sociedad que fue interpelada por esa literatura, a tener peculiaridades específicas en un momento del devenir histórico. Ya no se necesitaría entonces de la memorización de datos sino de la exploración de transiciones históricas que pueden llevar al lector a comparar entre si los diferentes pasados que aborda, así como también esos pasados con su situación actual.

Sumadoa esto, la posibilidad de historiar literaturas de áreas culturales específicas - llámense regiones, departamentos, unidades étnico culturales - puede vincular de manera más sólida a los estudiantes con su territorio más inmediato. Como ya se ha afirmado en este texto, no se trata de desconocer algunos hitos de la tradición colombiana, ni tampoco de afrontar el estudio de la literatura de una variante intranacional con un fervor irracional: la cuestión es ampliar el canon de obras para explorar aquellos escritores que desarrollaron un ejercicio de réplica frente a las realidades de un área cultural específica.

A partir de ese estudio, quienes hacen parte del mundo escolar - universitario y colegial - pueden poner a dialogar sus propios imaginarios - o por lo menos los que se han expresado en la literatura de su territorio más cercano - con las ideas y formas de expresión nacionales hispanoamericanas y universales. Hoy que se habla cada vez con mayor empeño y en ocasiones con esnobismo, sobre globalización y desterritorialización, en donde al parecer todos pertenecemos a la aldea global, es necesario volver sobre los elementos configuradores del individuo, es decir, a esas formas de vida y de expresión compartida con quienes se ha vivido una estrecha historia común.

Sólo en ese conocimiento de las especificidades territoriales que construyen un ser humano, se evita su disolución en la masa de mensajes que pueblan la sociedad contemporánea y se dialoga con mayor decisión e igualdad con las expresiones foráneas. No se trata de negar el conocimiento de otras formas de vida, $y$ de adoptar un hermetismo dogmático que preserve a un área cultural específica de los "demonios" externos; es más bien impulsar una visión de lo local para acceder a diálogos con los demás.

Para la enseñanza de la literatura colombiana esto puede significar la exploración de nuevas obras que, sin llegar a desconocer a nombres como Isaacs, Rivera o García Márquez, configuren la historia literaria de un área 
cultural específica. Hay que enfatizar en este sentido que estudiar los escritores de una determinada área cultural (o en su defecto de un departamento o región) no significa volver sobre las obras del regionalismo, en donde afloran tan solo los rasgos folclóricos de una sociedad, sino observar la variada gama de evaluaciones - algunas con bastos niveles de universalidad - que dichos escritores han hecho. Asumiendo esta actitud, se puede no sólo ampliar en el estudiante los nombres clásicos que rondan por su cabeza cada que se habla de literatura colombiana, sino también generar una inquietud por las historias locales, por los procesos estético literarios de su territorio más cercano.

Finalmente, las indagaciones en la historia de la lectura (de textos literarios), puede conjugarse con el estudio de las instituciones literarias para canalizar ejercicios críticos sobre ese otro componente en la conformación de la literatura: los lectores. Proyectos en historia de la lectura literaria en escuelas, colegios y universidades, o de historia de la crítica literaria (colombiana o de un área cultural específica) arrojarían resultados pertinentes para conocer los lastres y las virtudes en la formación de amantes de la literatura.

En este tipo de indagaciones es posible releer textos no literarios de gran valía - como revistas y periódicos culturales - en los cuales se ha ejercido una evaluación sobre múltiples obras con enfoques diversos. El estudiante se aproximaría así a un entendimiento más completo no sólo de las particularidades de lenguaje y cosmovisión de una obra, sino de los movimientos de recepción de textos contemporáneos o posteriores a una pieza artística específica.

Estas son algunas de las proyecciones de una nueva historia de la literatura colombiana que pretenda impactar en la enseñanza en varios niveles. Del entusiasmo con el que se asuman las nuevas discusiones en las universidades y aun en los colegios, depende que la efectividad de las propuestas se lleve a buen término y podamos en el futuro extirpar de nuestro vocabulario las quejas frente a la falta de interés de los estudiantes por la lectura, o sobre la poca producción interpretativa sobre la literatura nacional.

\section{BIBLIOGRAFÍA}

AA.VV. Literatura y cultura: narrativa colombiana del siglo XX. Colombia: Ministerio de Cultura, 2000

Acosta, Carmen Elisa. Leer literatura. Ensayos sobre la lectura literaria en el siglo XIX. Bogotá: Cooperativa Editorial Magisterio, 2005.

Teoría Historia Crítica

Bedoya Sánchez, Gustavo Adolfo. "La periodización de la literatura colombiana en sus historias literarias: tradición y rupturas" En JALLA. CD - ROM, 2006.

"Hacia una Nueva Historia de la Literatura Colombiana: balance teórico y metodológico" En XV Congreso de colombianistas. 2007. CD ROM.

D'Allemand, Patricia. "Rediseñando fronteras culturales:mapasalternativos paralahistoriografía literaria latinoamericana" En Literatura: teoría, historia, crítica. No 5, 2003, pp. 74-104

Giraldo, Luz Mary. La novela colombiana ante la crítica (1975-1990). Bogotá y Cali: Centro editorial Javeriano y Editorial de la facultad de humanidades de la U del Valle, 1994

Gutiérrez Girardot, Rafael. Horas de estudio. [s.l]: Biblioteca colombiana de Cultura, [s.f.]

"Literatura y Sociedad" En Carranza María Mercedes (Selección). Estravagario. Colombia: Instituto colombiano de Cultura, 1976.

Aproximaciones. Bogotá: Procultura, 1986.

El Intelectual y la historia. Caracas: Fondo Editorial La Nave Va, 2001.

Henao Restrepo, Darío. "Clásicos regionales. Una memoria indispensable para el futuro" En Poligramas. No 25, Julio de 2006. 
Patiño, Germán. "La pérdida del objeto. Historiografía literaria e historias de la literatura colombiana" En Poligramas. No 21, Junio de 2004, pp. 241 - 267.

Poppel, Hubert. "Literatura y comunicación: un intento de pensar la historia de la literatura desde Jauss, Habermas y Welsch" En Literatura: teoría, historia, crítica. No 5, 2003, p. 15

Rodríguez Luna, María Elvira. "Manuales de literatura y/o negación de lo literario" En Litterae. No 4, Agosto de 1991.

Trujillo Patricia. "Los comienzos de la novela colombiana en el siglo XX" En Jaramillo Zuluaga, Eduardo (Edición y Prólogo) Colombia: tiempos de imaginación y desafío. Colombia: Asociación de colombianistas, 2007.

Vallejo Murcia, Olga. "Las fuentes para el estudio historiográfico de la literatura colombiana y su aporte a una Nueva Historia de la literatura colombiana" XV Congreso de colombianistas. 2007. CD ROM.

"Sobre el papel de la historia literaria en la enseñanza de la literatura" En Papel Periódico (Revista Digital)

Vargas García, Nicolay. "Literaturas de minorías: presencia en el imaginario; ausencia en la historia" En JALLA. CD - ROM. 2006. 